\title{
An exploration of the use of personalised nutrition among potential end users of the concept
}

\author{
O. C. McMahon, Á. McConnon, K. McKenzie and E. Gibney \\ Institute of Food and Health, University College Dublin, Ireland
}

The burden of chronic diseases is rapidly increasing worldwide ${ }^{(1)}$. The top diseases share key risk factors of unhealthy diets, physical inactivity and alcohol use: all of which are closely related to obesity, type- 2 diabetes and the metabolic syndrome ${ }^{(2)}$. Inspired by the human genome project, nutritional genomics is said to have the potential to contribute to disease prevention and treatment in the future, with personalised nutrition as the theoretical application of nutritional genomics ${ }^{(3)}$.

Establishing current knowledge and attitudes of potential end-users early in the introduction of a new technology such as personalised nutrition serves to identify potential 'blind spots' regarding social, ethical, cost, practical and other implications, which must be addressed. Research among the general public in Europe has shown that individuals who have or perceive themselves to have high blood pressure, high blood cholesterol, central obesity or other risk factors for the metabolic syndrome were more likely to undergo genetic testing to receive a personalised $\operatorname{diet}^{(4)}$.

The current study aimed to qualitatively explore morbidly obese and type- 2 diabetes patient groups as potential targets for personalised nutrition testing in the future. Attitudes, openness and concerns about personalised nutrition were to be discussed with the groups. The study also aimed to investigate dieticians' attitudes and interest in integrating personalised nutrition into health care delivery, and the potential ability to do so based on the dieticians' own experiences. Focus groups were held with morbidly obese patients ( $n$ 10), type- 2 diabetes patients $(n 10)$ and dietitians $(n$ 8). Thematic analysis of the focus groups took place using the NVivo8 software.

Dietician groups were open to the concept of personalised nutrition in the clinical setting, but knowledge was low. From previous experience with patients and behaviour change, dieticians exhibited some doubt as to whether behaviour change in patients would be achieved, though the potential for motivation was well recognised. From their clinical experience, dieticians were unsure of the relevance of personalised nutrition to this setting as chronic diseases were not seen as a priority here according to the policies they worked under. Patient groups were very interested in the concept. However, they emphasised that although the potential for motivation would be high, behaviour change could not be guaranteed as they had tried to change and failed many times (factors such as free will and cravings were important in preventing change). Their past experiences with fad diets led them to explore potential concern with the source of any testing in the future. The importance of data protection was highlighted by patients from their previous experiences with discrimination.

The results draw attention to relevant issues that must be addressed in order for the concept of personalised nutrition to be incorporated successfully in the future. The results are indicative that morbidly obese and type- 2 diabetes clinical patients may be potential end-user targets for personalised nutrition testing as it develops further. It is also evident that dieticians desire education and scientific evidence in order to remain open to utilising the concept of personalised nutrition in the future.

1. World Health Organization (2002) Diet, Nutrition and the Prevention of Chronic Diseases: Report of a Joint WHO/FAO Expert Consultation. Geneva, Switzerland: World Health Organization.

2. World Health Organization (2002) The World Health Report 2002-Reducing Risks, Promoting Healthy Life. Geneva, Switzerland: World Health Organization.

3. Muller M \& Kersten S (2003). Nutrigenomics: goals and strategies. Nat Rev Genet 4, 315-322.

4. Stewart-Knox et al. (2009) Attitudes toward genetic testing and personalised nutrition in a representative sample of European consumers. Br J Nutr 101, 982-989. 